\title{
Classification of Surimi Gel Strength Patterns Using Backpropagation Neural Network and Principal Component Analysis
}

\author{
Krisana Chinnasarn ${ }^{1}$, David Leo Pyle ${ }^{2}$, and Sirima Chinnasarn ${ }^{3}$ \\ ${ }^{1}$ Department of Computer Science, Burapha University, Thailand \\ ${ }^{2}$ c/o School of Chemical Engineering and Analytical Science, \\ The University of Manchester, UK \\ ${ }^{3}$ Department of Food Science, Burapha University, Thailand
}

\begin{abstract}
This paper proposes two practically and efficiently supervised and unsupervised classifications for surimi gel strength patterns. An supervised learning method, backpropagation neural network with three layers of 17-34-4 neurons for each later, is used. An unsupervised classification method consists of the data dimensionality reduction step via the PCA algorithm and classification step using correlation coefficient similarity measure. In the similarity measure step, each surimi gel strength pattern is compared with the surimi eigen-gel patterns, produced by the PCA step. In this paper, we consider a datum pattern as a datum dimension. The training data sets (12 patterns or 12 data dimensions) of surimi gel strength are collected from 4 experiments having different fixed setting temperature at $35^{\circ} \mathrm{C}, 40^{\circ} \mathrm{C}, 45^{\circ} \mathrm{C}$, and $50^{\circ} \mathrm{C}$, respectively. Testing data sets (48 patterns) are including original training set and their added Gaussian noise with 1, 3 and 5 points, respectively. From the experiments, two proposed methods can classify all testing data sets into its proper class.
\end{abstract}

Keywords: Backpropagation neural network, Principal component analysis, surimi eigen-gel patterns, backprogpagation neural network.

\section{Introduction}

Surimi is a concentrate of the myofibrillar proteins, primarily myosin and actin, of fish muscle, that is mechanically deboned, water-washed fish meat. In order to manufacture surimi-based products, the raw or frozen surimi is ground with salt and other ingredients, and then passed through thermal processing to set the shape and develop the texture 8]. During the thermal process, myofibrillar protein is induced to form the actomyosin gel, mainly derived from the myosin portions, which gives the unique elastic textural properties of the products 44. Surimi is very well-known in food processing topics. Applications or surimi based products are Crap-analog or Kani-kamabogo, Kamaboko, Chikuwa, Satsumage, fish sausage and Shell fish-analog, etc. 8] 
Generally, surimi gel is formed following two heating-steps, a preheating process below $50^{\circ} \mathrm{C}$ (setting step) prior to cooking at $80^{\circ} \mathrm{C}$ or $90^{\circ} \mathrm{C}$ to produce an opaque, highly elastic and strengthened gel [8]. The setting period is an important step in surimi gel development. This is because the setting at low temperature prior to heating at a higher temperature allows slow ordering of the protein molecule resulting in good gelation, fine structure and great elasticity 6] 8]. Different temperatures in the setting step result in different conformation of the proteins leading to different structure, and strength of the final gel [7].

The qualities of surimi gel, notably gel strength and structure, can be affected during gelation by various physical conditions such as high pressure, heating temperature and heating period during setting. In this paper, we emphasize on only surimi gel strength patterns which obtained from various setting temperatures and times. From the preliminary experiments, it can be observed that all patterns of surimi gel strength have similar shape. The final gel strengths increased to the maximum value and then decreased to the plateau in all setting tempertures. It is difficult to determine classes of each surimi gel strenth. In order to determine their proper classes, both supervised and unsupervised classification learning methods are applied to identify the qualities of surimi gel pattern.

The paper is organized as follows: Section 2 explains Cod surimi material, backpropagation neural network, principal surimi gel strength construction, and similarity method; Some experimental results are presented in Section 3 and conclusions in Section 4, respectively.

\section{Materials and Methods}

\section{$2.1 \quad$ Materials}

Cod (Gadus morhua) fillets were purchased from Frosts Fish LTD, Reading, UK. The fillets were skinned, rinsed with clean water, blended in a Lab Micronizer (Waring Commercial), washed by the ratio of water: minced fish at $3: 1(\mathrm{v} / \mathrm{w})$. Then, the minced fish was dewatered by a basket centrifuge, then mixed with $6 \%$ sugar and $0.2 \%$ tetrasodium pyrophosphate and stored in an air blast freezer at $-18^{\circ} \mathrm{C}$ as a frozen surimi sample.

\subsection{Gel Preparation}

The frozen surimi was thawed at $4^{\circ} \mathrm{C}$ until the temperature of surimi reached $0^{\circ} \mathrm{C}$ and then, mixed with $2.5 \%$ salt by using a Lab Micronizer (Waring Commercial). The sol obtained was stuffed into stainless-steel cylinders of $2.5 \mathrm{~cm}$. inner diameter and $2.5 \mathrm{~cm}$. length. Surimi gels were prepared by heat setting at $35^{\circ} \mathrm{C}, 40^{\circ} \mathrm{C}, 45^{\circ} \mathrm{C}$ and $50^{\circ} \mathrm{C}$ for $0,5,10,15,20,25,30,35,40,45,50,55,60,120$, 180,240 , and 300 minutes in a water bath (Grant Y28, type VFP). Then, the gels were cooked at $90^{\circ} \mathrm{C}$ for 20 minutes, and cooled in ice water. The obtained surimi gels were stored at $4^{\circ} \mathrm{C}$ for 24 hours before analysed. 


\subsection{Gel Strength Analysis}

The gel strengths of surimi gels were tested by using a TA-XT2 Texture Analyser (Stable Micro Systems Ltd., Surrey, UK.). All the cooked gels were compressed to $15 \mathrm{~mm}$ at the speed of $1.1 \mathrm{~mm} /$ second using a cylindrical shaped probe of $2.5 \mathrm{~cm}$ in diameter. Then, the changes in applied force were recorded. The gel strength was obtained from the peak force multiplied by the compression distance at the peak.

\subsection{Proposed Classification Methods}

The objective of the paper is to classify each surimi gel strength pattern into a proper class. We propose both supervised and unsupervised learning methods to identify the class or quality of surimi gel patterns.

Backpropagation Neural Network. Artificial neural network or neural network is a parallel processing mechanism which similar to human brain learning system. There are both supervised, unsupervised and reinforcement learning systems [5. The first and simple neural network structure is called the Perceptron neural network. Perceptron consists of input neurons, connection weight, adding function, activation function, and output as illustrated in Fig. 1(a).

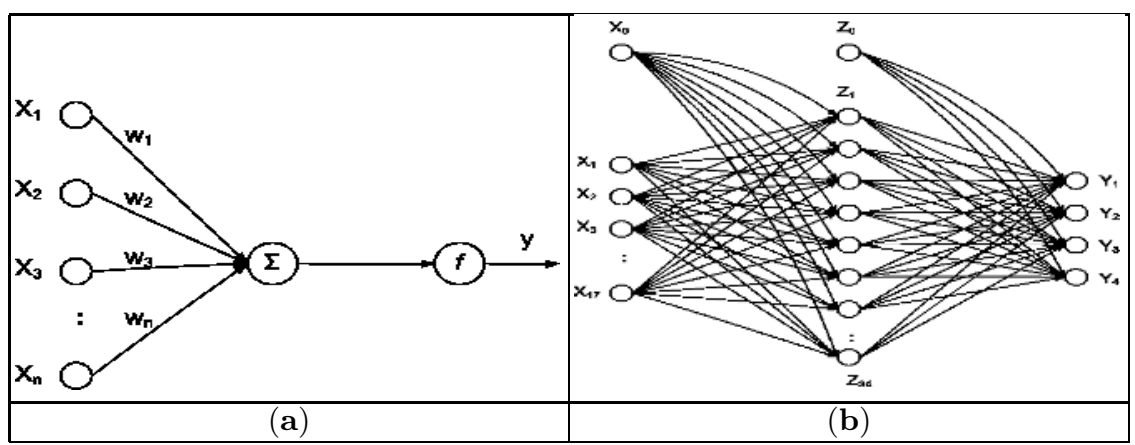

Fig. 1. (a) Perceptron neural network and (b) Backpropagation neural network used in the paper

where $\left[x_{1}, x_{2}, \cdots, x_{n}\right]^{T}$ are the input vectors, $\left[w_{1}, w_{2}, \cdots, w_{n}\right]^{T}$ are the connection weights, $\beta$ is a bias, $f()$ is an activation function and $y$ is an output. $y$ can be written as follows:

$$
\begin{aligned}
n e t & =\sum\left(x_{1} w_{1}+x_{2} w_{2}+\cdots+x_{n} w_{n}\right)+\beta \\
y & =f(n e t)
\end{aligned}
$$


The backpropagation neural network [5] with three layers, input-hidden- output, is used. The neurons in each layer are 17-34-4 for input-hidden-output layer, respectively. Neural network learning algorithm starts with the random weight $w_{h i}$ and $w_{j h}$. The input vectors $\left[x_{1}, x_{2}, \cdots, x_{17}\right]^{T}$ are fed to the hidden layer $Z$ using the weight $w_{h i}$. Then hidden vectors $\left[z_{1}, z_{2}, \cdots, z_{34}\right]^{T}$ are fed again by the weight $w_{j h}$ in order to produce the output vectors $\left[y_{1}, \cdots, y_{4}\right]^{T}$. Backpropagation network structure is shown in Fig. 1(b). Let $t$ be a desired target. The objective function is a summed squared error (SSE) as follows:

$$
E=\frac{1}{2} \sum(y-t)^{2}
$$

And the activation function is a Sigmoidal function as follows:

$$
f(n e t)=\frac{1}{1+e^{-n e t}}
$$

Principal Component Analysis to Surimi Gel Pattern. The second method for classifying each surimi gel strength is the Principal component analysis and the similarity measure method. An identifiable system consists of two main parts, the construction of surimi gel eigen-patterns and similarity analysis. First, the PCA method is used for searching an eigen-pattern by returning the principal direction for each class of surimi gel quality. Second, correlation coefficient similarity method between the eigen gel pattern and surimi gel strength pattern is used for classifying a class of them. A structure chart of the overall system is illustrated in Figure 2 .

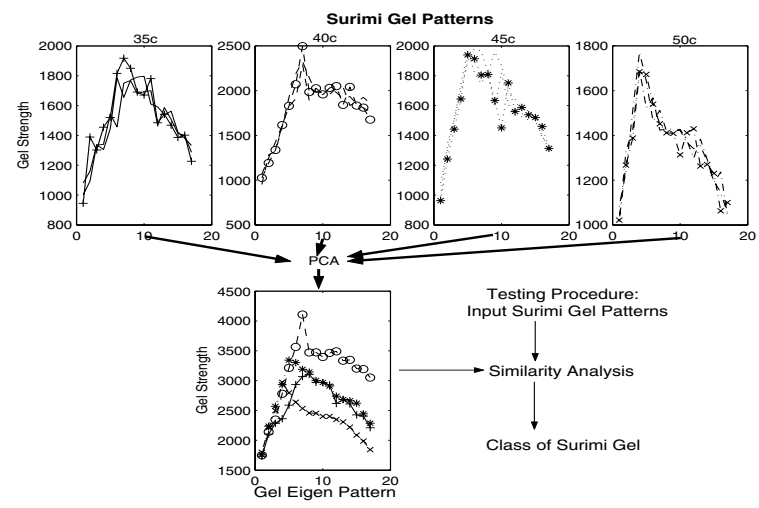

Fig. 2. System Structures for Surimi Gel Pattern Identification

Constructing principal direction or eigen-pattern is presented in this section 2. Constructing procedures are composed of two main steps, surimi gel strength matrix formation and computing an eigen-pattern. First, the surimi gel strength matrix formation step, input data for modeling surimi gel eigen-patterns are 
surimi gel strength from 3 replications, detailed in Section 2.2. Then from the previous conditions, three input vectors of surimi gel strength were obtained for each setting temperature. And they are formed as a row major matrix with a dimension of 3 by 17 .

Second, computing an eigen-pattern step, the principal component analysis can be carried out. The calculation of surimi gel eigen-pattern is given by the solution to the following step [3]:

1. Compute the mean for each row mean $_{i}$

2. Generate the zero-mean matrix $\mathbf{M z}$ for each row $i$,

$$
\mathbf{M z}_{i, j}=\mathbf{M}_{i, j}-\text { mean }_{i}
$$

where $1 \leq i \leq 17$.

3. Compute the covariance matrix $\mathbf{C o v}$ for $\mathbf{M z}$,

$$
\operatorname{Cov}(\mathbf{M z})=\mathbf{E}\left[(\mathbf{M z})(\mathbf{M z})^{T}\right]
$$

where $\mathbf{E}[$.$] is an expectation value and (\mathbf{M z})^{T}$ is a transpose matrix of $\mathbf{M z}$. Then the dimensions of $\mathbf{C o v}(\mathbf{M z})$ are 3 by 3 .

4. Compute an eigenvalue, $d$, and eigenvector, $v$, for the covariance matrix Cov.

5. Project $\mathbf{M}$ onto new axis by eigen-vector $v$,

$$
\mathbf{P}=v^{T} \mathbf{M}
$$

where $v^{T}$ is a transpose matrix of $v$

Similarity Measure. The main objective of the classification problem is to find a natural grouping in a set of data [3. In other words, we want to say that the samples in class $c_{i}$ are more like one another than like samples in other class $c_{j}$, where $i \neq j$.

Correlation Coefficient Analysis, $\rho$. The correlation coefficient $\rho$ between two random variable $x$ and $y$ can be described as:

$$
\rho(x, y)=\frac{\operatorname{cov}(x, y)}{\sqrt{\operatorname{var}(x) \operatorname{var}(y)}}
$$

where $\operatorname{cov}(x, y)$ is the covariance between two random variables and $\operatorname{var}($.$) is$ the variance of a random variable. Correlation coefficient values are within $[-1, \ldots, 1]$. If $x$ and $y$ are completely correlated, $\rho(x, y)$ is 1 or -1 . If they are absolutely uncorrelated, $\rho(x, y)$ is 0 .

\section{Experimental Results}

Some simulations have been made on surimi Gel Strength patterns, which contain 17 data points for each pattern, details were given in Section 2.2. Testing sets (12 data) include the original training set and their added Gaussian noise with 1, 3 and 5 points, respectively. Then, in total, the testing sets have 48 patterns. Graphical representation of all testing sets are illustrated in 


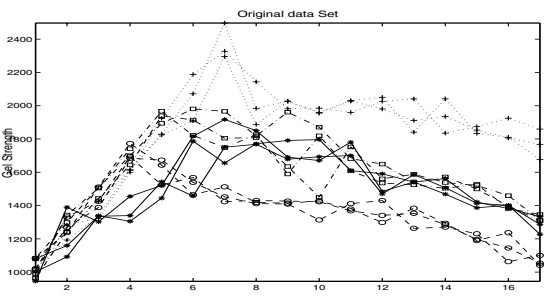

(a)

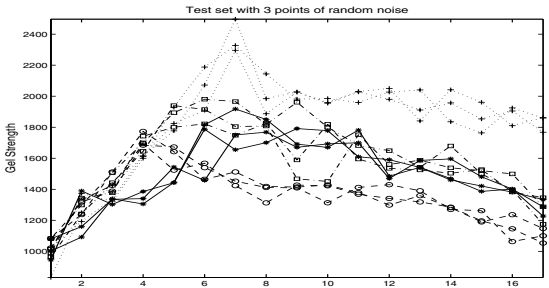

(c)

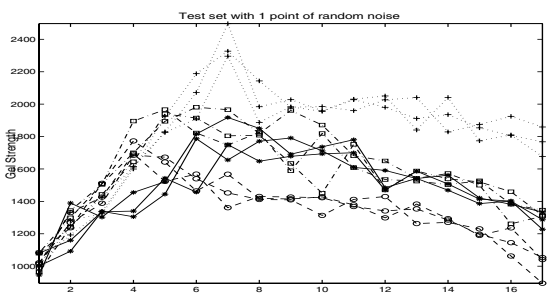

(b)

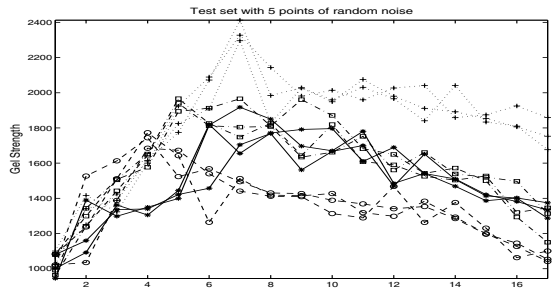

(d)

Fig. 3. (a) Original surimi gel patterns created by $35^{\circ} \mathrm{C}, 40^{\circ} \mathrm{C}, 45^{\circ} \mathrm{C}$ and $50^{\circ} \mathrm{C}$, (b) Original surimi gel added one point of Gaussign noise, (c) Original surimi gel added three points of Gaussign noise and (d) Original surimi gel added five points of Gaussign noise

Figure 3(a), (b), (c), and (d), respectively. It can be seen that it is very difficult to identify a class for input pattern by hand. How can we do that?

\subsection{Backpropagation Neural Network}

In the learning step, we set $\epsilon \leq 0.00001$. The learning is terminated after 35 epochs. Tables 1(a), (b), (c), and (d) display the probability of each surimi gel strength pattern to be each expected class. Probability values from Tables 1(a), (b), (c), and (d) confirm that all testing set patterns can be classified into a proper class even they have some noises. For example from Table 1(d), degree of similarity between testing pattern 1 to expected class 1 , class 2 , class 3 , and class 4 are $0.9957,0.0000,0.0000$, and 0.0000, respectively. Then, it is classified to class 1 which is correct.

\subsection{Principal Component Analysis}

Tables $2(\mathbf{a}),(\mathbf{b}),(\mathbf{c})$ and $(\mathbf{d})$ display the degree of similarity between testing data sets and the principal direction of surimi gel strength using correlation coefficient analysis. Degree of similarity among them from Tables 2(a), (b), (c) and (d) confirm that all testing set patterns can be classifed into a proper class even they have some noises. For example from Table 2 (c), degree of similarity between testing pattern 3 to expected class 1 , class 2 , class 3 , and class 4 are $0.9462,0.8789,0.7765$, and 0.4154 , respectively. Then it is classified to class 1 which is correct. 
Table 1. Probability of the surimi gel strength pattern using backpropagation neural network (a) original surimi gel patterns, (b) added 1 point of random gaussian noise, (c) added 3 points of random gaussian noise and (d) added 5 points of random gaussian noise

\begin{tabular}{|c|c|c|c|c|c|c|c|c|c|}
\hline \multirow{2}{*}{$\begin{array}{l}\text { Testing } \\
\text { pattern }\end{array}$} & \multicolumn{4}{|c|}{ Expected classes } & \multicolumn{4}{|c|}{ Expected classes } & \multirow{2}{*}{$\begin{array}{c}\text { Its Actual } \\
\text { class }\end{array}$} \\
\hline & Class 1 & Class 2 & Class 3 & Class 4 & Class 1 & Class 2 & Class 3 & Class 4 & \\
\hline 1 & 0.9998 & 0.0000 & 0.0000 & 0.0000 & 0.9999 & 0.0000 & 0.0000 & 0.0000 & 1 \\
\hline$\overline{2}$ & 0.9999 & 0.0013 & 0.0000 & 0.0000 & 0.9998 & 0.0015 & 0.0000 & 0.0000 & 1 \\
\hline 3 & 1.0000 & 0.0000 & 0.0000 & 0.0000 & 1.0000 & 0.0000 & 0.0000 & 0.0000 & 1 \\
\hline 4 & 0.0000 & 0.9994 & 0.0008 & 0.0000 & 0.0000 & 0.9994 & 0.0008 & 0.0000 & 2 \\
\hline 5 & 0.0001 & 0.9996 & 0.0000 & 0.0000 & 0.0001 & 0.9996 & 0.0000 & 0.0000 & 2 \\
\hline 6 & 0.0000 & 0.9984 & 0.0001 & 0.0000 & 0.0000 & 0.9814 & 0.0029 & 0.0000 & $\overline{2}$ \\
\hline 7 & 0.0000 & 0.0000 & 0.9995 & 0.0000 & 0.0000 & 0.0000 & 0.9998 & 0.0000 & 3 \\
\hline 8 & 0.0000 & 0.0000 & 0.9999 & 0.0000 & 0.0000 & 0.0000 & 0.9999 & 0.0000 & $\overline{3}$ \\
\hline$\overline{9}$ & 0.0000 & 0.0008 & 0.9990 & 0.0000 & 0.0000 & 0.0008 & 0.9990 & 0.0000 & $\overline{3}$ \\
\hline 10 & 0.0000 & 0.0000 & 0.0002 & 0.9999 & 0.0000 & 0.0000 & 0.2592 & 0.9930 & 4 \\
\hline 11 & 0.0000 & 0.0000 & 0.0005 & 1.0000 & 0.0000 & 0.0000 & 0.0011 & 1.0000 & 4 \\
\hline \multirow[t]{2}{*}{12} & 0.0002 & 0.0000 & 0.0000 & 1.0000 & 0.0000 & 0.0000 & 0.0248 & 1.0000 & 4 \\
\hline & \multicolumn{4}{|c|}{$(\mathbf{a})$} & \multicolumn{4}{|c|}{ (b) } & \\
\hline 1 & $\overline{0.9989}$ & 0.0000 & 0.0000 & 0.0000 & $\overline{0.9957}$ & 0.0000 & 0.0000 & 0.0000 & 1 \\
\hline 2 & 0.9643 & 0.0000 & 0.0000 & 0.0000 & 1.0000 & 0.0001 & 0.0000 & 0.0000 & 1 \\
\hline 3 & 1.0000 & 0.0000 & 0.0000 & 0.0000 & 1.0000 & 0.0000 & 0.0000 & 0.0000 & 1 \\
\hline 4 & 0.0000 & 0.9992 & 0.0056 & 0.0000 & 0.0000 & 0.9960 & 0.0000 & 0.0000 & 2 \\
\hline 5 & 0.0000 & 0.9997 & 0.0000 & 0.0000 & 0.0000 & 0.9996 & 0.0000 & 0.0000 & 2 \\
\hline 6 & 0.0000 & 0.9984 & 0.0002 & 0.0000 & 0.0000 & 0.9988 & 0.0001 & 0.0000 & 2 \\
\hline 7 & 0.0002 & 0.0074 & 0.9984 & 0.0000 & 0.0000 & 0.0000 & 0.9992 & 0.0000 & 3 \\
\hline 8 & 0.0000 & 0.0000 & 1.0000 & 0.0000 & 0.0000 & 0.0000 & 0.9995 & 0.0000 & 3 \\
\hline 9 & 0.0000 & 0.0005 & 0.9991 & 0.0000 & 0.0000 & 0.0005 & 0.9972 & 0.0000 & 3 \\
\hline 10 & 0.0004 & 0.0000 & 0.0000 & 0.9995 & 0.0000 & 0.0000 & 0.0351 & 1.0000 & 4 \\
\hline 11 & 0.0000 & 0.0000 & 0.0000 & 1.0000 & 0.0000 & 0.0000 & 0.0000 & 1.0000 & 4 \\
\hline \multirow[t]{2}{*}{12} & 0.0002 & 0.0000 & 0.0001 & 0.9999 & 0.0000 & 0.0000 & 0.0027 & 1.0000 & 4 \\
\hline & \multicolumn{4}{|c|}{$(\mathbf{c})$} & \multicolumn{4}{|c|}{$(\mathbf{d})$} & \\
\hline
\end{tabular}

Table 2. Correlation coefficient $(\rho)$ between (a) original surimi gel patterns, (b) added 1 point of random gaussian noise, (c) added 3 points of random gaussian noise and (d) added 5 points of random gaussian noise and the principal surimi gel eigen-patterns

\begin{tabular}{|c|c|c|c|c|c|c|c|c|c|}
\hline \multirow{2}{*}{$\begin{array}{l}\text { Testing } \\
\text { pattern }\end{array}$} & \multicolumn{4}{|c|}{ Expected classes } & \multicolumn{4}{|c|}{ Expected classes } & \multirow{2}{*}{$\begin{array}{c}\text { Its Actual } \\
\text { class }\end{array}$} \\
\hline & \begin{tabular}{|l|} 
Class 1 \\
\end{tabular} & Class 2 & Class 3 & Class 4 & Class 1 & Class 2 & Class 3 & Class 4 & \\
\hline 1 & 0.9575 & 0.8661 & 0.7968 & 0.4126 & 0.9572 & 0.8421 & 0.8006 & 0.4142 & 1 \\
\hline 2 & 0.9585 & 0.8258 & 0.8662 & 0.5848 & 0.9581 & 0.8264 & 0.8761 & 0.5949 & 1 \\
\hline 3 & 0.9534 & 0.8567 & 0.7852 & 0.4321 & 0.9449 & 0.8334 & \begin{tabular}{|l|l|}
0.7677 \\
\end{tabular} & 0.4182 & 1 \\
\hline 4 & 0.8717 & 0.9805 & 0.7685 & 0.3218 & 0.8722 & 0.9806 & 0.7691 & 0.3227 & 2 \\
\hline 5 & 0.8807 & 0.9896 & 0.7594 & 0.3489 & 0.8806 & 0.9898 & 0.7635 & 0.3531 & 2 \\
\hline 6 & 0.8678 & 0.9894 & 0.7307 & 0.3105 & 0.8706 & 0.9857 & 0.7365 & 0.3229 & 2 \\
\hline 7 & 0.7763 & \begin{tabular}{|l|}
0.6666 \\
\end{tabular} & 0.9659 & 0.8288 & 0.7266 & 0.5869 & 0.9246 & 0.8297 & 3 \\
\hline 8 & 0.8874 & 0.7663 & 0.9653 & 0.7679 & 0.8893 & 0.7673 & 0.9657 & \begin{tabular}{|l|l|}
0.7679 \\
\end{tabular} & 3 \\
\hline 9 & 0.7992 & 0.7661 & \begin{tabular}{|l|}
0.9549 \\
\end{tabular} & 0.7524 & 0.7993 & 0.7670 & 0.9549 & \begin{tabular}{|l|}
0.7524 \\
\end{tabular} & 3 \\
\hline 10 & 0.5423 & 0.3323 & 0.8013 & 0.9819 & 0.5730 & 0.3794 & 0.8212 & 0.9741 & 4 \\
\hline 11 & 0.4523 & 0.2820 & 0.7529 & 0.9717 & 0.4556 & 0.2649 & 0.7368 & 0.9587 & 4 \\
\hline \multirow[t]{2}{*}{12} & 0.4919 & 0.3613 & 0.8201 & 0.9713 & 0.4353 & 0.2792 & 0.7715 & 0.9652 & 4 \\
\hline & \multicolumn{4}{|c|}{ (a) } & \multicolumn{4}{|c|}{$(\mathbf{b})$} & \\
\hline 1 & 0.9565 & 0.8656 & 0.8003 & 0.4076 & 0.9425 & 0.8575 & 0.7916 & 0.4205 & 1 \\
\hline 2 & 0.9659 & 0.8174 & 0.8384 & 0.5187 & 0.9501 & 0.8440 & 0.7805 & 0.4328 & 1 \\
\hline 3 & 0.9462 & 0.8789 & 0.7765 & 0.4154 & 0.9338 & 0.8443 & 0.7172 & 0.3690 & 1 \\
\hline 4 & 0.8617 & 0.9751 & 0.7702 & 0.3253 & \begin{tabular}{|l|}
0.8698 \\
\end{tabular} & 0.9777 & 0.7382 & 0.2794 & 2 \\
\hline 5 & 0.8993 & 0.9875 & 0.7875 & 0.3741 & 0.9005 & 0.9853 & 0.7622 & 0.3499 & 2 \\
\hline 6 & 0.8635 & 0.9848 & 0.6980 & 0.2759 & 0.8597 & 0.9826 & 0.7261 & 0.3158 & 2 \\
\hline 7 & 0.6937 & \begin{tabular}{|l}
0.6066 \\
\end{tabular} & 0.9313 & 0.8130 & 0.8094 & 0.6932 & 0.9713 & 0.8010 & 3 \\
\hline 8 & 0.8871 & 0.7564 & 0.9542 & 0.7791 & 0.8875 & 0.7346 & 0.9383 & 0.7427 & 3 \\
\hline 9 & 0.8061 & 0.7788 & 0.9432 & 0.7446 & 0.8568 & 0.8045 & 0.9708 & \begin{tabular}{|l|l|}
0.7488 \\
\end{tabular} & 3 \\
\hline 10 & 0.5288 & 0.3145 & 0.7921 & 0.9635 & 0.3667 & 0.1332 & 0.6376 & 0.9196 & 4 \\
\hline 11 & 0.4302 & 0.2484 & 0.7185 & 0.9584 & 0.3782 & 0.2118 & 0.6627 & 0.9181 & 4 \\
\hline \multirow[t]{2}{*}{12} & 0.4894 & 0.4065 & 0.8076 & 0.9281 & \begin{tabular}{|l}
0.5128 \\
\end{tabular} & 0.4503 & 0.8260 & 0.9138 & 4 \\
\hline & \multicolumn{4}{|c|}{ (c) } & \multicolumn{4}{|c|}{ (d) } & \\
\hline
\end{tabular}

\section{Concluding Remarks}

This paper reports some applications of Backpropagation neural network and PCA (Principal Component Analysis). Backpropagation neural network is the 
supervised learning method. But PCA is an unsupervised classification method based on data information. Throughout the paper, the identification of surimi gel patterns using Backpropagation neural network and eigen-pattern together with the correlation coefficient has been outlined. Good identification is obtained from the Backpropagation neural network but we need to know a class of each pattern in advance. On the other hand, the advantages of the PCA method developed in this paper are as follows. (1) Each class or principal direction of surimi gel strengths is obtained by an unsupervised method, the PCA. Thus, for further identification of the surimi gel strength class, we can easily do this by passing surimi gel strength into the PCA. Then a new class of surimi gel strengths is created. In other words, the principal direction of surimi gel strengths is dynamic based on data characteristics. (2) It can be concluded that the correlation coefficient method gives an efficient result in the case where the testing patterns have very similar shape.

\section{References}

1. Chen.T, Amari.S.-I. and Lin.Q. A Unified Algorithm for Pricipal and Minor Component Extraction Neural Networks, Vol. 11, pp. 385-390, 1998.

2. Chinnasarn.K, Chinnasarn.S, and Pyle.D.L., Surimi Gel Pattern Identification Using Eigen-Pattern and Similarity Analysis, accepted to the $9^{\text {th }}$ National Computer Science and Engineering Conference: NCSEC2005, Bangkok, Thailand.

3. Duda.R.O, Hart.P.E., and Stork.D.G. Pattern Classification, $2^{\text {nd }}$ edition, John Wiley \& Sons, Inc., 2001.

4. Hall, G.M. and Ahmad,N.H. Surimi and fish-mince products. In G.M. Hall (ed). Fish Processing Technology. London: Blackie Acadimic \& Professional. 1997.

5. Haykin.S. Neural Network a Comprehensive foundation. 2nd, Prentice Hall,1999.

6. Hermansson, A.M. Physico-chemical aspects of soy proteins structure formation. Journal of Texture Studies. 9: pp 33-58, 1978.

7. Hossain, M.I., et al. Contribution of the polymerization of protein by disulfide bonding to increased Gel Strength of walleye pollack surimi gel with preheating time. Fisheries Science. 67 : pp 710-717, 2001.

8. Lanier, T.C. and Lee, C.M. Surimi Technology. New York : Marcel Dekker, 1992. 